\title{
Which one's more work? Predicting effective credit hours between courses
}

\author{
Shruthi Chockkalingam \\ University of California, Berkeley \\ shruthi_chock@berkeley.edu
}

\author{
Run Yu \\ New York University \\ run.yu@nyu.edu
}

\author{
Zachary A. Pardos \\ University of California, Berkeley \\ pardos@berkeley.edu
}

\begin{abstract}
University students select courses for an upcoming term in part based on expected workload. Course credit hours is often the only metric given by the institution relevant to how much work a course will be and does not serve as a precise estimate due to the lack of granularity of the metric which can lead to student under or overestimation. We define a novel task of predicting relative effective course credit hours, or time load; essentially, determining which courses take more time than others. For this task, we draw from institutional data sources including course catalog descriptions, student enrollment histories and ratings from a popular course rating website. To validate this work, we design a personalized survey for university students to collect ground truth labels, presenting them with pairs of courses they had taken and asking which course took more time per week on average. We evaluate which sources of data using which machine representation techniques provide the best prediction of these course time load ratings. We establish a benchmark accuracy of 0.71 on this novel task and find skip-grams applied to enrollment data (i.e., course2vec), not catalog descriptions, to be most useful in predicting the time demands of a course.
\end{abstract}

\section{CCS CONCEPTS}

\section{- Applied computing $\rightarrow$ Education.}

\section{KEYWORDS}

course load, time load, course2vec, enrollment data, higher education, predictive analytics, institutional data, survey study

\section{ACM Reference Format:}

Shruthi Chockkalingam, Run Yu, and Zachary A. Pardos. 2021. Which one's more work? Predicting effective credit hours between courses. In LAK21: 11th International Learning Analytics and Knowledge Conference (LAK21), April 12-16, 2021, Irvine, CA, USA. ACM, New York, NY, USA, 7 pages. https://doi.org/10.1145/3448139.3448204

\section{INTRODUCTION}

When selecting courses for one term of a university degree program, students are faced with a dilemma: what combination of courses will challenge them and keep them engaged without giving

Permission to make digital or hard copies of all or part of this work for personal or classroom use is granted without fee provided that copies are not made or distributed for profit or commercial advantage and that copies bear this notice and the full citation on the first page. Copyrights for components of this work owned by others than the author(s) must be honored. Abstracting with credit is permitted. To copy otherwise, or republish, to post on servers or to redistribute to lists, requires prior specific permission and/or a fee. Request permissions from permissions@acm.org.

LAK21, April 12-16, 2021, Irvine, CA, USA

(C) 2021 Copyright held by the owner/author(s). Publication rights licensed to ACM. ACM ISBN 978-1-4503-8935-8/21/04 ..\$15.00

https://doi.org/10.1145/3448139.3448204 them an unmanageable workload? Usually, they can look to a few different indicators for some clues: the credit hour designation by instructors for each course in their planned schedule, advice from other students, and grade distributions from previous offerings of courses they are considering. Each of these, though, has its own issues. Credit hour designations are imperfect and coarse-grained and therefore not all $\mathrm{n}$-credit hour courses are equal in terms of hours of work required per week. Advice from other students is anecdotal and not necessarily representative of the typical student. Finally, average grades can also be misleading because the grade does not always correspond to the amount of time required and many courses grade on a curve, obscuring the raw percentage score. This points to the need for a "course load metric" that can more accurately and precisely determine which courses require a greater amount of work to succeed. With this more fine-grained information in-hand, students could better compose their term's set of courses and avoid severe over or under estimation of workload that could result in reduced learning outcomes.

A useful notion of "course load" is one that derives from the psychological concept of mental workload. One way of measuring mental workload is through the Subjective Workload Assessment Technique (SWAT), a procedure that determines the workload associated with a task based on three factors [15]. The first is time load, or the amount of time a worker has to accomplish the task. The second is mental effort load, which is the amount of concentration needed to complete the task. The final criterion is psychological stress load, or the internal and external factors (e.g., anxiety, frustration, noise in the surrounding environment) that pose a barrier to finishing the task. Course load can be seen as a combination of all three of these factors applied to a course as a whole rather than one particular task: the amount of time the course demands, the amount of concentration it requires, and the amount of confusion and other negative feelings it provokes.

In this paper, we focus on the time load aspect of course load. Course load in education literature has typically been used synonymously with the number of credit hours for a course, which are meant to be a measure of time $[1,9,19]$. Credit hours are designed as an estimate of the number of hours per week a student is expected to spend on a course. However, credit hours are not fine-grained, only providing a rough estimate of the time requiredone 3-credit hour course could take significantly more time than another. In addition, credit hour ratings can also be inaccurate; a newly established course rated at 2 credit hours can in practice end up taking the same amount of time as a 4-credit hour course. Therefore, there is a need to determine the true or effective credit hours associated with a course, or what we call its course time load.

Various institutional sources of data about courses were gathered to aid our time load predictive model. These sources included course 
catalog descriptions, student enrollment histories, and ratings from a popular course ratings website. We then created vector representations from these data sources to correspond to each course. Using these vectors, we attempted to predict which of two presented courses had the higher time load, with ground truth labels collected from student responses from a survey designed for this study. We took two approaches to making this pair-wise prediction. The first approach attempted to treat predicting existing, imperfect course credit hour ratings as a regression task, regularized by the institutional course data vectors. When making the pairwise prediction, the course which has the higher credit hours prediction would be selected. The second approach was a direct supervised training of models to predict the student labelled higher time load course.

\section{RELATED WORK}

Course time load is a previously unstudied measure in the field of learning analytics and educational data mining. More attention has been recently paid to other institutional tasks, such as predicting students' course enrollments, grades, and prerequisites.

Of the three tasks mentioned above, grade prediction has been most prevalent in the literature. Elbadrawy and Karypis [4] applied collaborative filtering to the grade prediction task with the goal of helping students select suitable courses. Ren et al. [17, 18] also attempted grade prediction by analyzing historical educational records, such as co-taken courses. Hu and Rangwala [6] presented two types of Bayesian deep learning models for grade prediction under a course-specific framework. Morsy and Karypis [10] analyzed the relationship between students' degree plans and their graduation GPA based on a dataset that spanned 16 years. Finally, Jiang et al. [8] employed an LSTM-based model to predict student letter and pass/fail grades.

Enrollment prediction (i.e., predicting which course a student will take next) has been a secondary area of research focus. [4] investigated how academic features such as students' majors and academic levels influence enrollment patterns. In order to implement academic early warning systems and help underperforming students get back on track, Brown et al. [2] used student grades and enrollment histories to make enrollment predictions. With data on student enrollment histories, course catalog descriptions, and course grades, [12] developed a personalized course guidance system using neural networks, suggesting courses based on predicted enrollments. Polyzou et al. [14] proposed a random-walk-based approach to capture the sequence relationships between different courses to recommend course lists to students.

A third area of institutional prediction tasks relates to inferring properties of courses such as prerequisite relationships and latent topics. Jiang and Pardos [7] applied Course2vec [12] and graph embedding techniques to predict prerequisites. $\mathrm{Hu}$ and Rangwala [5] used course grades and student enrollment data to predict prerequisite chains. Finally, enrollment data have been used to infer latent keywords and topics of courses Dong et al. [3], Motz et al. [11].

Jiang and Pardos [7] provide a summary of additional related work in this quickly developing area. The various successes highlighted above using institutional data and neural embeddings to learn latent aspects of courses suggest that they may carry useful signals for predicting course load features, which our study is concerned with predicting.

\section{COLLECTING GROUND TRUTH TIME LOAD DATA}

In order to validate our models, which aspire to accurately predict relative time load, we needed a ground truth data source for higher quality labels. However, existing data sources, such as course credit hours and data from learning management systems (LMS), only serve as an inexact proxy for time load. Credit hours, as previously discussed, are not granular enough. LMS clickstream data can provide details about the number of assignments, late assignments, and activity within the LMS, but not how much time is spent on these assignments outside of the LMS. Therefore, we went to students to collect ground truth labels in the form of a personalized survey, taken on an existing course recommendation platform with access to enrollment histories.

We designed the survey to ask the following question: "Below are two courses you received the same grade in. Which course took you more hours per week to achieve that grade?" We asked students to make pairwise comparisons, rather than absolute rankings or ratings, because we anticipated that students' recollections about exactly how much time a course took may be fuzzy. We also anticipated that students may bias their rating of time load based on the grade they received, favoring the lower grade course as having higher time load. To avoid this potential bias, we asked students to only compare courses in which they received the exact same letter grade (e.g. B+ grade courses compared to other $\mathrm{B}+$ grades rather than compared to B- grades). We also only asked students to compare courses in which they receive a $\mathrm{C}$ - or above. This was due to our assumption that grades below this point, which include failing grades, would contain too much variability in time spent to receive those grades and thus would likely not produce reliable pairwise ratings. We limited the survey to juniors and seniors to ensure that participants would have a course history long enough to make several comparisons between courses they received the same grade in. If students did not have at least ten pairs of courses they could compare, they were excluded from participating in the survey. If they had more than twenty pairs they could compare, twenty of the possible pairs were presented to them at random. On average, students had 19 pairs of courses to rate.

In order to present students with course pairs from their history, we engineered a survey interface into an existing course recommender system platform ${ }^{1}$ at UC Berkeley, a public university. Figure 1 displays the interface of the survey study. This system was implemented using a Python Flask back-end and Angular front-end, with a simple MySQL database. Table 1 shows an example of a single user course rating collected in the study.

Juniors and seniors from the University were invited to participate through various popular Facebook groups associated with the University. We also sent emails to a number of students who had participated in past studies and expressed interest in future studies. We had $n=56$ participants, with each participant being given a $\$ 10$ gift card to a popular e-commerce website to complete the survey.

$\overline{{ }^{1} \text { https://askoski.berkeley.edu }}$ 
Overall, 436 courses were presented to be rated by students in our survey, totalling 1,058 pairwise time load ratings. The quality of the responses was assessed by analyzing submission times. For a single survey response, the mean submission time was $10.89 \mathrm{~s}$, the median $4.71 \mathrm{~s}$, and minimum $0.76 \mathrm{~s}$. Six were submitted in under $1 \mathrm{~s}$. Based on these statistics, we chose not to exclude any responses. Of the 1,058 course pairs, there were 44 that were duplicates. Of these, 15 were rated oppositely by the two students who rated them.

Table 1: Example student rating from the survey log data

\begin{tabular}{|l|l|}
\hline anon_id: 123456 & course_a: Journalism_122 \\
\hline course_b: Geography_149A & course_selected: Geography_149A \\
\hline duration: 12.818 & timestamp: 1589214177.4140222 \\
\hline
\end{tabular}

\section{DATASETS}

In addition to the ground truth data collected from the user study, we worked with several other datasets, described in this section.

\subsection{Course Catalog Description Data}

We used an API from the University to retrieve course titles and catalog descriptions. We retrieve these data for a total of 9,986 courses, which covered $99 \%$ of the courses students rated in the time load survey. Table 2 shows an example of course information we collected from this API. Figure 2 shows a histogram of credit hours for these nearly 10 thousand courses, which was an additional feature returned by the API.

Table 2: An example of course catalog description data

course_id: DATA_144 1 course_title: Data Mining \& Analytics

course_description: This course introduces students to practical fundamentals of data mining and machine learning with just enough theory to aid intuition building. The course is projectoriented, with a project beginning in class every week and to be completed outside of class by the following week, or two weeks for longer assignments. The in-class portion of the project is meant to be collaborative, with the instructor working closely with groups to understand the learning objectives and help them work through any logistics that may be slowing them down. Weekly lectures introduce the concepts and algorithms which will be used in the upcoming project. Students leave the class with hands-on data mining and data engineering skills they can confidently apply.

\subsection{Student Enrollment Data}

We used a dataset from the same institution as the students in the survey, which consists of anonymized student course enrollment data from Fall 2008 to Spring 2017. Each row of the dataset contains an anonymized student ID, course name and number, semester year, and the grade the student received in the course. The dataset contains enrollment histories of 80,503 students with 1,984,422 total enrollments. There were 7,941 courses in all that were enrolled in, all with catalog description data from the API, and covering $98 \%$ of the courses rated in the time load survey. After filtering out courses that were not found in both the course catalog description data and the student enrollment data, we were left with $\mathrm{N}=431$ courses and a total of 1,017 ratings from the survey. This was the validation set used for all experiments except for those involving "NinjaCourses" data, described in the next section.

\subsection{NinjaCourses Data}

NinjaCourses is a website where students can quickly plan their schedule and see student ratings and reviews of courses and instructors. It was developed by members of the University community; however, it is not affiliated with the University. Though NinjaCourses is no longer being updated as of the Fall 2016 semester, it remains a popular source of information that students consult. Ratings on NinjaCourses are expressed across four dimensions: course assignments, course exams, instructor helpfulness, and instructor enthusiasm. Additionally, some student ratings include comments that detail their experience in the course. We collected NinjaCourses ratings and comments posted between Fall 2007 to Spring 2016 for all courses that students had rated in our time load survey. Of the 436 courses rated in the survey, 318 courses (or $73 \%$ ) were found in our data collected from the NinjaCourses website. On average, these 318 courses each had 72 student ratings, of which 40 included text comments. The data format is shown in Table 3. After filtering out ratings from our own survey of courses that were not found in the NinjaCourses dataset, we were left with $\mathrm{N}=295$ courses and a total of 687 ratings from the survey. Only the experiments involving NinjaCourses ratings and comments used this reduced validation set.

\section{EFFECTIVE CREDIT HOUR PREDICTION METHODOLOGY}

Using various course representation vectors, discussed in Section 6, we designed two different modeling approaches to predicting course time load. The first, credit hour regression, attempted to use course vectors and campus supplied credit hours to predict the relative difference in credit hours between courses. The second, pairwise classification, was trained to directly classify which of the two input course vectors would be rated as having a higher time load.

\subsection{Credit Hour Regression}

In this method, we use official credit hours as a proxy for time load and do not utilize our time load user study validation data during training. The training of this method can therefore be seen as unsupervised with respect to the target being predicted (i.e., pair-wise selections in our time load validation data). The rationale behind this approach was that the regression might learn a correlation between high and low credit hour courses and course features from the embedding. In the best case, the embedding as input to the credit hour prediction model might serve as useful regularization and add granularity, since the model would produce a continuous credit hour prediction instead of being limited to an integer between one and four. 
Progress: 3 / 20

Below are two courses you received the same grade in. Which course took you more hours per week to achieve that grade?

\section{COMPSCI 70: Discrete Mathematics and Probability Theory \\ Subject: Computer Science \\ Instructors: Chung-wei Lin \\ You took this course in: Summer 2015}

Catalog Description: Logic, infinity, and induction; applications include undecidability and stable marriage problem. Modular arithmetic and GCDs; applications include primality testing and cryptography. Polynomials; examples include error correcting codes and interpolation. Probability including sample spaces, independence, random variables, law of large numbers; examples include load balancing, existence arguments, Bayesian inference.

\section{This One}

\section{COMPSCI 160: User Interface Design and}

\section{Development}

Subject: Computer Science

Instructors: Eric J Paulos

You took this course in: Spring 2016

Catalog Description: The design, implementation, and evaluation of user interfaces. User-centered design and task analysis. Conceptual models and interface metaphors. Usability inspection and evaluation methods. Analysis of user study data. Input methods (keyboard, pointing, touch, tangible) and input models. Visual design principles. Interface prototyping and implementation methodologies and tools. Students will develop a user interface for a specific task and target user group in teams.

Figure 1: Screenshot from the student survey. A student is shown two courses from their history and asked which took more time to achieve the same grade.

Table 3: An example of Ninja Courses data for one course

\begin{tabular}{|l|l|l|l|}
\hline course_id & Computer Science_189 & average_rating & 76 \\
\hline average_assignments_rating & 7 & average_exams_rating & 66 \\
\hline average_helpfulness_rating & 70 & average_enthusiasm_rating & 72 \\
\hline $\begin{array}{l}\text { comments: ['Loved Malik, focused on the derivation of various conc ept and went into depth (not as much as other classes, but that's } \\
\text { alright). He was engaging, and really fun to listen to and learn from. Easy class overall, tests were easy and assignments were fun.'] }\end{array}$ \\
\hline
\end{tabular}

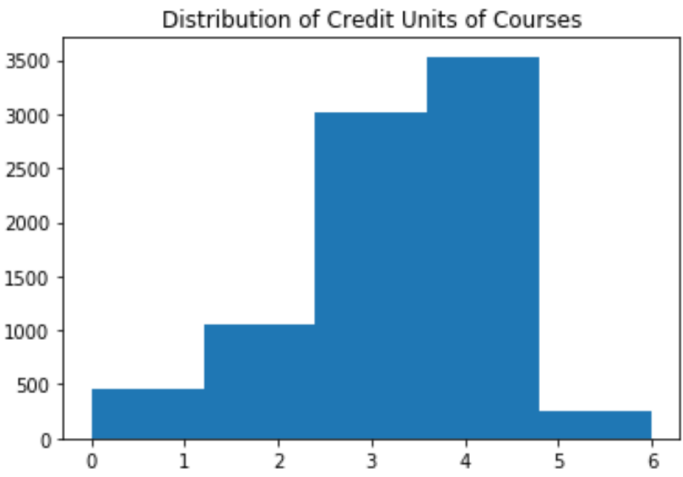

Figure 2: Histogram of credit hours

Methods used to predict the official credit hours of the input course included "credit hour regression" and "credit hour neural network." In credit hour regression, a linear regression was performed from a set of vectors representing each course, to the number of official credit hours each course was worth. If the predicted credit hours of course A was higher than the predicted credit hours of course B, we predicted that course A has a greater time load than course B. The credit hour neural network method used a single 20-node hidden layer neural network to perform the same task. In these ranking methods, we have used credit hours as a proxy for course time load. The accuracy of each of these methods was determined by calculating the percentage of correctly predicted selections between two courses in our validation survey data.

\subsection{Pairwise Classification Method}

In this method, we utilized our collected time load validation data to train models to directly predict which of the two input courses were chosen by a student as having the higher effective course time load. Figure 3 depicts this course classification approach. Methods used for classification included "validation set logistic regression" and "validation set neural network." For both, 10 -fold cross validation was performed to determine the accuracy of the method. The input to the models was a vector representation of course A concatenated with a representation of course $B$. The output label was 0 if course A was selected as higher time load in the survey and 1 if not. The neural network classifier used a simple 20-node hidden layer feedforward neural network with logistic output activation. For our best performing vector set (with the 20-node $\mathrm{NN}$ ), we also tried a 100-node neural network and a random forest (RF), which did not improve accuracy. 


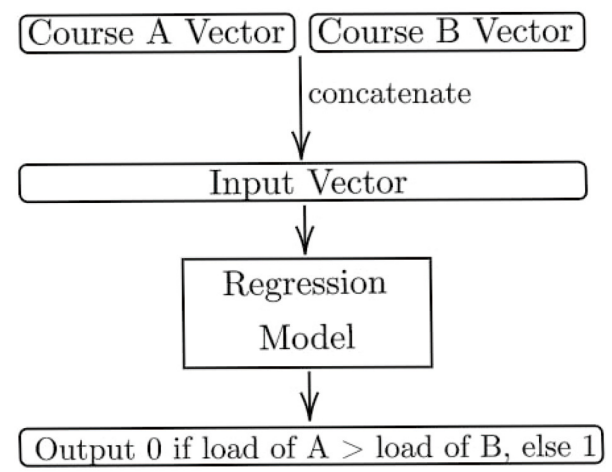

Figure 3: Direct pairwise classification method diagram

\section{REPRESENTATION METHODS}

To aid in the novel task of predicting relative time load between pairs of courses, we evaluated different ways of representing courses as vectors, based on the data sources discussed in Section 3 .

\subsection{Course2Vector}

Course vectors learned from enrollment histories were taken from previous research [13]. Inspired by the applications to natural language, this method frames a student enrollment sequence as a "sentence", and courses within the sequence as "words." To be more specific, for each student, a chronological course enrollment sequence is produced by first sorting by semester then randomly serializing within-semester course order. Then, a skip-gram model is applied to these sequences to learn course vector embeddings. In language models, two word vectors are typically cosine similar if they share similar sentence contexts. Likewise, in the education domain, if courses share many student co-enrollments, they will likely be close to one another in the vector space.

For this research, we used course2vec vectors that performed best in an analogy validation task from Pardos and Jiang [13], which involved evaluating how well the vector space completed 2,256 pre-specified analogies such as, Math $1 \mathrm{~B}$ is to Honors Math $1 \mathrm{~B}$ as Physics 7B is to Honors Physics 7B.

\subsection{Bag-of-Words}

For each course, its NinjaCourses comments were concatenated and vectorized. The vectorization method used for the comments was bag-of-words (unigram).

Each NinjaCourses course vector was created by aggregating student ratings for that course; for example, if the average ratings for a course in the categories "Overall", "Assignments", "Exams", "Enthusiasm", and "Helpfulness" were 78, 85, 86, 63, and 65 respectively, and the students' comments are concatenated into a single string, then the course vector representation would be $[78,85,86$, $63,65]$ concatenated with the vector representation of the comment string.

\subsection{Sentence BERT}

Sentence-BERT (SBERT) [16] is a modification of the pretrained BERT network to derive semantically meaningful sentence embeddings that can be compared using cosine-similarity. After preprocessing (such as removing punctuation and stop words), we applied SBERT to course catalog descriptions which returned vectors of length 768 .

\section{RESULTS}

For each model tested, we assigned an accuracy score. The score for a particular model is determined by what percent of the 1,017 pairwise comparisons of course time load from the survey the model predicted correctly. That is, the percentage of comparisons in which the model and student agree on the course that takes more time to receive the same grade in. The results are summarized in the table 4.

Overall, methods using the student enrollment data performed the best, with both the neural network and the random forest trained on course 2 vec vectors resulting in an accuracy of $71 \%$. Methods involving credit hour prediction all scored under $60 \%$ accuracy. Of all credit hour prediction methods, the neural network based on SentenceBERT was the most promising at 59\% accuracy. Ensemble methods combining all other classifiers together were also tried, and surprisingly, this resulted in a degradation of accuracy. This could be due to highly correlated predictions between course $2 \mathrm{vec}$ and SBERT, as we calculated that the two models overlap in $86 \%$ of the instances.

Results confirm that official credit hours are not a useful proxy for time load and that the expected regularization of the course vectors did not help. NinjaCourses ratings were not effective, perhaps because the NinjaCourses information is outdated, or because student comments on the site are not only about courses but also past instructors' teaching styles. It is also plausible that students' perception of a course's goodness in the NinjaCourse areas of "Assignments", "Exams", "Enthusiasm," "Helpfulness," and "Overall” are not closely related to the time load demand of the course.

Interestingly, student enrollment-based representation (i.e., course2vec) performed best at predicting relative time load. Perhaps because it provides information on which courses students typically take later into their academic careers that may be more 
Table 4: Accuracy of different methods and data sources on the pairwise classification problem

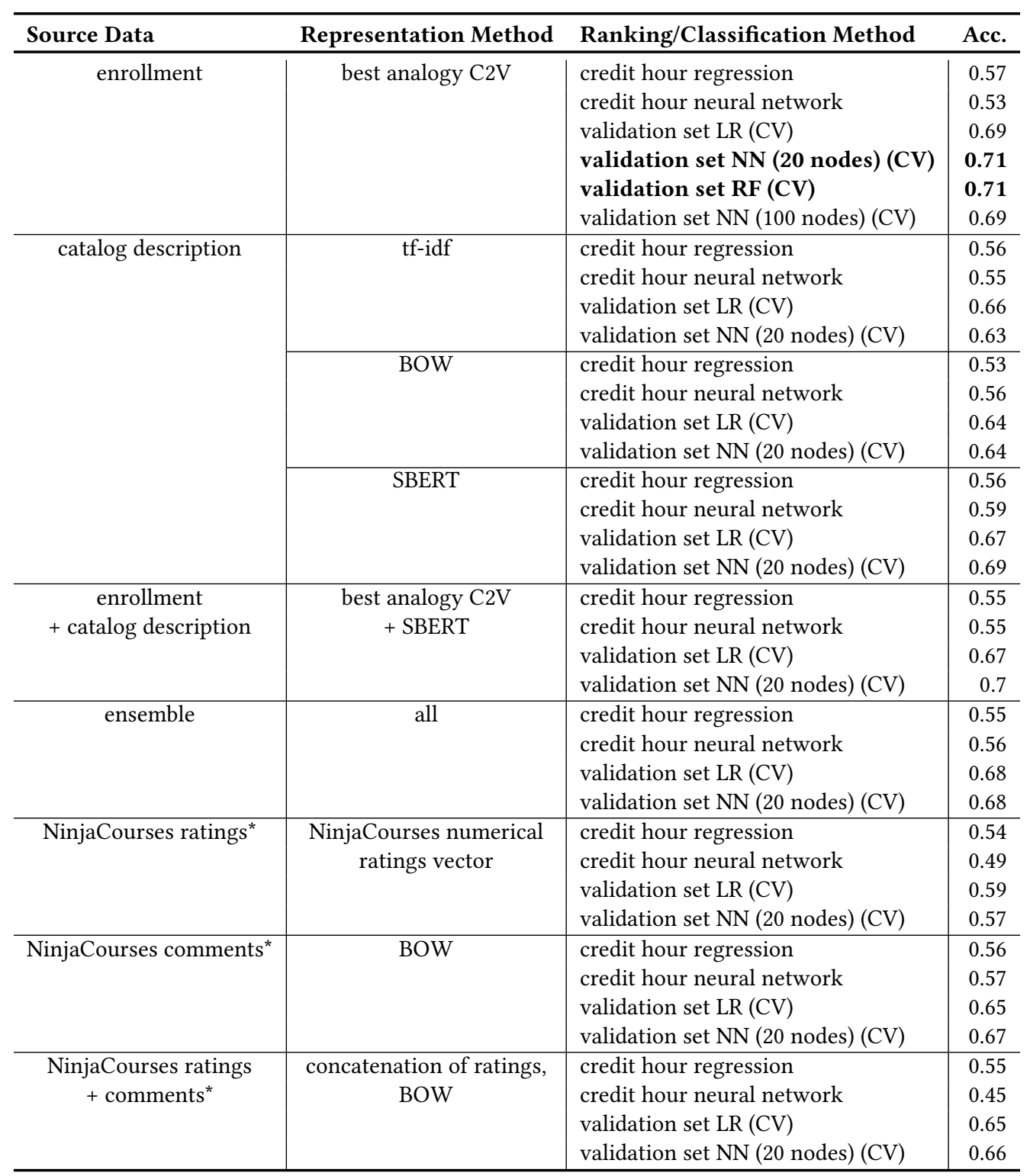

*NinjaCourses ratings were not available for all courses students rated (318 of 436 courses used for these experiments)

demanding and time intensive and which students avoid taking together because of their high time demand.

\section{DISCUSSION}

In this work, we introduced the task of predicting effective relative course time load. We conducted a series of prediction experiments using enrollments, catalog descriptions, and externally sourced course comments and ratings as input to several models. We found the most useful source of data for this task to be course enrollment history, modeled using course2vec. This approach achieved an accuracy of 0.71 in predicting students' pairwise time load ratings between courses they had taken before and received the same grade in. Slightly less effective were course catalog descriptions, which performed best when modeled using sentence-BERT (0.69 acc). Surprisingly, the combination of sentence-BERT and Course2Vec representations did not lead to an overall improvement, scoring 0.70 acc. Externally sourced course comments from students performed considerably better than externally sourced course ratings ( $0.67 \mathrm{vs}$ $0.59 \mathrm{acc}$ ), but neither improved overall accuracy when included in an ensemble of representations ( $0.68 \mathrm{acc})$.

Our analyses confirmed that official course credit hours were not useful in predicting effective course time load and thus are an imprecise and insufficient indicator to use to compare which course has a higher time demand. This finding should motivate the need for 
a better measure that students can consult when selecting courses and for further research to be conducted on this topic in order to improve the accuracy of these measures from the benchmarks established in this work.

\section{LIMITATIONS AND FUTURE WORK}

There were several limitations in our approach. Our time load survey was not diverse with respect to student major, with students in STEM comprising $91 \%$ of respondents, with $27 \%$ of them majoring in mechanical engineering. It may be found that there is a different proportional benefit of catalog description in non-STEM courses, for example. A limitation of our general method is that we aim to predict a single time load per course. While this is commonplace, given courses have a single course credit hours attribute, expected time load likely varies by the background preparation of the individual and thus a more personalized approach to time load prediction could be explored. While this work focused on time load, future work could explore predicting other factors of course load, such as mental effort and psychological stress. Lastly, our methodology made predictions of pairwise ratings of course time load (i.e., which course had greater load). Future work could seek to predict a numeric load rating on a scale that could be compared across many courses.

\section{REFERENCES}

[1] Clive Belfield, Paul Davis Jenkins, and Hana Elizabeth Lahr. 2016. Momentum: The Academic and Economic Value of a 15-Credit First-Semester Course Load for College Students in Tennessee (2016)

[2] Michael Geoffrey Brown, R Matthew DeMonbrun, and Stephanie D Teasley. 2018. Conceptualizing co-enrollment: Accounting for student experiences across the curriculum. In Proceedings of the 8th International Conference on Learning Analytics and Knowledge. 305-309.

[3] Matthew Dong, Run Yu, and Zachary A Pardos. 2019. Design and Deployment of a Better Course Search Tool: Inferring Latent Keywords from Enrollment Networks. In European Conference on Technology Enhanced Learning. Springer 480-494.

[4] Asmaa Elbadrawy and George Karypis. 2016. Domain-aware grade prediction and top- $n$ course recommendation. In Proceedings of the 10th ACM Conference on Recommender Systems. 183-190.

[5] Qian Hu and Huzefa Rangwala. 2019. Academic Performance Estimation with Attention-based Graph Convolutional Networks. arXiv preprint arXiv:2001.00632 (2019).

[6] Qian Hu and Huzefa Rangwala. 2019. Reliable deep grade prediction with uncertainty estimation. In Proceedings of the 9th International Conference on Learning Analytics \& Knowledge. 76-85.

[7] Weijie Jiang and Zachary A Pardos. 2020. Evaluating Sources of Course Information and Models of Representation on a Variety of Institutional Prediction Tasks. International Educational Data Mining Society (2020).

[8] Weijie Jiang, Zachary A Pardos, and Qiang Wei. 2019. Goal-based course recommendation. In Proceedings of the 9th International Conference on Learning Analytics \& Knowledge. 36-45.

[9] Zakir Khouj, Abdullah Mohammad, Ahmed Shami, and Mohammad Ansar. 1982 Relationship of Students' Course Loads with their Grade Point Average Scores, Study II: An Occasional Paper. (1982).

[10] Sara Morsy and George Karypis. 2019. A Study on curriculum planning and its relationship with graduation GPA and time to degree. In Proceedings of the 9th International Conference on Learning Analytics \& Knowledge. 26-35.

[11] Benjamin Motz, Thomas Busey, Martin Rickert, and David Landy. 2018. Finding Topics in Enrollment Data. In Proceedings of the 11th International Conference on Educational Data Mining.

[12] Zachary A Pardos, Zihao Fan, and Weijie Jiang. 2019. Connectionist recommendation in the wild: on the utility and scrutability of neural networks for personalized course guidance. User Modeling and User-Adapted Interaction 29, 2 (2019), 487-525.

[13] Zachary A Pardos and Weijie Jiang. 2020. Designing for serendipity in a university course recommendation system. In Proceedings of the Tenth International Conference on Learning Analytics \& Knowledge. 350-359.
[14] Agoritsa Polyzou, Athanasios N Nikolakopoulos, and George Karypis. 2019. Scholars Walk: A Markov Chain Framework for Course Recommendation. International Educational Data Mining Society (2019).

[15] Gary B. Reid and Thomas E. Nygren. 1988. The Subjective Workload Assessment Technique: A Scaling Procedure for Measuring Mental Workload. Advances in Psychology Human Mental Workload (1988), 185-218. https://doi.org/10.1016/ s0166-4115(08)62387-0

[16] Nils Reimers and Iryna Gurevych. 2019. Sentence-bert: Sentence embeddings using siamese bert-networks. arXiv preprint arXiv:1908.10084 (2019).

[17] Zhiyun Ren, Xia Ning, Andrew S Lan, and Huzefa Rangwala. 2019. Grade Prediction Based on Cumulative Knowledge and Co-Taken Courses. International Educational Data Mining Society (2019).

[18] Zhiyun Ren, Xia Ning, and Huzefa Rangwala. 2017. Grade prediction with temporal course-wise influence. arXiv preprint arXiv:1709.05433 (2017).

[19] Sujit S. Sansgiry and Kavita Sail. 2006. Effect of Students Perceptions of Course Load on Test Anxiety. American fournal of Pharmaceutical Education 70, 2 (2006), 26. https://doi.org/10.5688/aj700226 